\title{
Bayesian Curve-Fitting in Semiparametric Small Area Models with Measurement Errors
}

\author{
Jinseub Hwang ${ }^{a}$, Dal Ho Kim ${ }^{1, b}$ \\ ${ }^{a}$ National Evidence-based Healthcare Collaborating Agency, Korea; \\ ${ }^{b}$ Department of Statistics, Kyungpook National University, Korea
}

\begin{abstract}
We study a semiparametric Bayesian approach to small area estimation under a nested error linear regression model with area level covariate subject to measurement error. Consideration is given to radial basis functions for the regression spline and knots on a grid of equally spaced sample quantiles of covariate with measurement errors in the nested error linear regression model setup. We conduct a hierarchical Bayesian structural measurement error model for small areas and prove the propriety of the joint posterior based on a given hierarchical Bayesian framework since some priors are defined non-informative improper priors that uses Markov Chain Monte Carlo methods to fit it. Our methodology is illustrated using numerical examples to compare possible models based on model adequacy criteria; in addition, analysis is conducted based on real data.
\end{abstract}

Keywords: Hierarchical Bayesian, nested error regression, penalized spline, radial basis, semiparametric, small areas, structural measurement

\section{Introduction}

Sample surveys are designed to provide estimates of totals and means of items of interest for large subpopulations (or domains). These estimates are "direct" because they use only domain-specific data with domain sample sizes large enough to support reliable direct "design-based" estimates. However, it is seldom possible to procure a large enough sample size to support direct estimates for small local areas such as counties, and school districts. The term "small area" to denote any domain for which direct estimates of adequate precision cannot be produced due to a small domain-specific sample size. It is often necessary to employ "indirect" estimates for small areas that can increase the "effective" domain sample size by "borrowing strength" from related areas through linking models and using some auxiliary data associated with the small areas. A comprehensive account of model-based small area estimation is given by Rao (2003).

Census and administrative data are often taken for auxiliary data in small area estimation with the assumption that auxiliary data are available for all areas and are measured without error. However, sometimes we can use auxiliary data from another survey and in this case the auxiliary data may be measured with error. This measurements error complicates the statistical analysis and this problem is commonly called measurement error problem (Fuller, 1987). This paper considers the situation in which auxiliary data are available for use in small area estimation, but where auxiliary data may be measured with error.

\footnotetext{
${ }^{1}$ Corresponding author: Department of Statistics, Kyungpook National University, 80 Daehakro, Bukgu, Daegu 702-701, Korea. E-mail: dalkim@knu.ac.kr
}

Published 31 July 2015 / journal homepage: http://csam.or.kr

(c) 2015 The Korean Statistical Society, and Korean International Statistical Society. All rights reserved. 
Semiparametric regression methods have not been used in small area estimation contexts until recently. This was mainly due to methodological difficulties in combining different smoothing techniques with estimation tools generally used in small area estimation. The pioneering contribution was due to work by Opsomer et al. (2008) that combined small area random effects with a smooth nonparametrically specified trend using penalized splines. Bhadra et al. (2013) considered a Bayesian semiparametric modeling procedure that used penalized splines to estimate the median household income for all of the United States.

In our previous model (Hwang and Kim, 2010), we developed a Bayesian semiparametric model to deal with the small area problem with measurement errors. We considered penalized splines for non-linear pattern that uses truncated polynomial basis functions (TPBF) with knots of equally spaced sample quantiles under a structural measurement error model. It follows from Hwang and Kim (2010) that the semiparametric model was superior to the model without nonparametric component (Ghosh et al., 2006).

This paper studies a Bayesian semiparametric model with radial basis functions (RBF) under a structural measurement error model for the small area problem with measurement errors. TPBF are not always numerically stable when the number of knots is large and the smoothing parameter is close to zero. Instead of TPBF, B-splines and RBF can deal with this problem (Ruppert et al., 2003). To fit our model and estimate parameters, we have conducted a hierarchical Bayesian (HB) approach with a Markov Chain Monte Carlo (MCMC) methodology that uses a Gibbs sampling and MetropolisHastings (M-H) algorithm.

We begin with a brief overview of the model specification and our notations in Section 2. In Section 3, we prove the propriety of the posterior and established the MCMC implementation of the proposed hierarchical Bayes procedure. In Section 4, we exhibit the performance of the proposed model based on simulation studies. We have checked the convergence and model adequacy using a potential scale reduction factor (PSRF) and the posterior predictive $p$-value (Meng, 1994); in addition, we also used root mean squared errors to compare models. We conduct the analysis based on real data in Section 5 and Section 6 discusses possible extensions of our modeling.

\section{Model Specification and Notations}

We consider a nested error regression model setup to estimate small area means originally considered by Battese et al. (1988). Suppose there are $m$ strata and each strata consists of the known population size $N_{i}$. Let $\left(y_{i j}, X_{i j}\right)$ denote the observed response and covariate of the $j^{\text {th }}$ unit in the $i^{\text {th }}$ stratum $\left(j=1, \ldots, N_{i} ; i=1, \ldots, m\right)$, respectively. The basis model can be expressed as

$$
y_{i j}=f\left(x_{i}\right)+u_{i}+e_{i j},
$$

where $u_{i}$ and $e_{i j}$ are the random effects and sampling errors with independent and identically distributed (i.i.d.) normal random variables, respectively. Here $f\left(x_{i}\right)$ is a smoothing function of $x_{i}$ reflecting the unknown response-covariate relationship; in addition, we use penalized splines with RBF. $\mathrm{RBF}$ is defined as

$$
1, x, \ldots, x^{p-1},\left|x-\tau_{1}\right|^{2 p-1}, \ldots,\left|x-\tau_{K}\right|^{2 p-1},
$$

where $|\cdot|$ is the function of absolute value. Here $K$ is the number of knots and we called this as the tuning parameter in our model. And $\tau=\left(\tau_{1}, \ldots, \tau_{K}\right)^{T}$ is the vector of knot locations on a grid equally spaced sample quantiles of covariate $x_{i}\left(\tau_{1}<\cdots<\tau_{K}\right)$. In our paper we consider $p=1$ case, then 
we can rewrite (2.1) as:

$$
y_{i j}=b_{0}+b_{1} x_{i}+\sum_{k=1}^{K}\left|x_{i}-\tau_{k}\right|+u_{i}+e_{i j} .
$$

Our superpopulation model with structural measurement error covariates is assumed as:

$$
\begin{aligned}
y_{i j} & =\boldsymbol{x}_{i}^{T} \boldsymbol{b}+\boldsymbol{z}_{i}^{T} \boldsymbol{\gamma}+u_{i}+e_{i j} \\
& =\theta_{i}+e_{i j} \\
X_{i j} & =x_{i}+\eta_{i j},
\end{aligned}
$$

where $\boldsymbol{x}_{i}=\left(1, x_{i}\right)^{T}, \boldsymbol{z}=\left(\left|x_{i}-\tau_{1}\right|, \ldots,\left|x_{i}-\tau_{K}\right|\right)^{T}$ and $\eta_{i j}$ is the measurement error with i.i.d. normal distribution. We consider structural measurement error model, so let $x_{i}$ is a normal random variable. Here $\boldsymbol{b}=\left(b_{0}, b_{1}\right)^{T}$ and $\boldsymbol{\gamma}=\left(\gamma_{1}, \ldots, \gamma_{K}\right)^{T}$ are the vector of regression and spline coefficients, respectively. Specifically, $\theta_{i}\left(=\boldsymbol{x}_{i}^{T} \boldsymbol{b}+\boldsymbol{z}_{i}^{T} \boldsymbol{\gamma}+u_{i}\right)$ is the small area means that we want to estimate. Finally, we assumed that $x_{i}, u_{i}, e_{i j}$ and $\eta_{i j}$ are mutually independent with $x_{i} \sim N\left(\mu, \sigma_{x}^{2}\right), u_{i} \sim N\left(0, \sigma_{u}^{2}\right)$, $e_{i j} \sim N\left(0, \sigma_{e}^{2}\right)$ and $\eta_{i j} \sim N\left(0, \sigma_{\eta}^{2}\right)$, respectively.

\section{Hierarchical Bayesian Estimation Procedure}

\subsection{Hierarchical Bayesian framework}

To fit the model and estimate parameters based on sample $\left(n_{i}\right.$ is drawn from the $i^{\text {th }}$ stratum and $\sum_{i=1}^{m} n_{i}=n_{t}$ ), we conduct a hierarchical Bayesian framework based on (2.2):

Stage 1. $y_{i j}=\theta_{i}+e_{i j}\left(j=1, \ldots, n_{i} ; i=1, \ldots, m\right)$

Stage 2. $\theta_{i}=\boldsymbol{x}_{i}^{T} \boldsymbol{b}+\boldsymbol{z}_{i}^{T} \boldsymbol{\gamma}+u_{i}(i=1, \ldots, m)$

Stage 3. $X_{i j}=x_{i}+\eta_{i j}\left(j=1, \ldots, n_{i} ; i=1, \ldots, m\right)$

Stage 4. $x_{i} \sim N\left(\mu_{x}, \sigma_{x}^{2}\right)$.

Stage 5. $\gamma \sim N\left(0, \sigma_{\gamma}^{2} I\right)$.

Stage 6. $b_{0}, b_{1}, \mu_{x}, \sigma_{e}^{2}, \sigma_{u}^{2}, \sigma_{x}^{2}, \sigma_{\eta}^{2}$ and $\sigma_{\gamma}^{2}$ are mutually independent with $b_{0}, b_{1}$ and $\mu_{x}$ i.i.d. uniform $(-\infty, \infty) ;\left(\sigma_{e}^{2}\right)^{-1} \sim G\left(a_{e}, b_{e}\right),\left(\sigma_{u}^{2}\right)^{-1} \sim G\left(a_{u}, b_{u}\right),\left(\sigma_{\eta}^{2}\right)^{-1} \sim G\left(a_{\eta}, b_{\eta}\right),\left(\sigma_{\gamma}^{2}\right)^{-1} \sim G\left(a_{\gamma}, b_{\gamma}\right)$, $\left(\sigma_{x}^{2}\right)^{-1} \sim G\left(a_{x}, b_{x}\right)$, where $G(\alpha, \beta)$ denotes a gamma distribution shape parameter $\alpha$ and rate parameter $\beta$ having the expression $f(x) \propto x^{\alpha-1} \exp (-\beta x)$.

\subsection{Propriety of the joint posterior}

Before the implementation, we check the propriety of the joint posterior based on given a hierarchical Bayesian framework since some priors are defined non-informative improper priors for regression parameters $b_{0}$ and $b_{1}$, and mean parameter $\mu_{x}$ of $x_{i}$. We can factorize the full posterior by conditional independence properties as

$$
\begin{aligned}
& {\left[\boldsymbol{\theta}, \boldsymbol{b}, \boldsymbol{\gamma}, \boldsymbol{x}, \mu_{x}, \sigma_{x}^{2}, \sigma_{e}^{2}, \sigma_{u}^{2}, \sigma_{\eta}^{2}, \sigma_{\gamma}^{2} \mid \mathbf{X}, \mathbf{y}\right]} \\
& \propto\left[\mathbf{y} \mid \boldsymbol{\theta}, \sigma_{e}^{2}\right]\left[\boldsymbol{\theta} \mid \boldsymbol{b}, \boldsymbol{\gamma}, \boldsymbol{x}, \sigma_{u}^{2}, \mathbf{X}\right]\left[\mathbf{X} \mid \boldsymbol{x}, \sigma_{\eta}^{2}\right]\left[\boldsymbol{x} \mid \mu_{x}, \sigma_{x}^{2}\right]\left[\boldsymbol{\gamma} \mid \sigma_{\gamma}^{2}\right][\boldsymbol{b}]\left[\mu_{x}\right]\left[\sigma_{x}^{2}\right]\left[\sigma_{e}^{2}\right]\left[\sigma_{u}^{2}\right]\left[\sigma_{\eta}^{2}\right]\left[\sigma_{\gamma}^{2}\right] .
\end{aligned}
$$


Theorem 1. Assume that $a_{e}, a_{u}, a_{x}, a_{\eta}$ and $a_{\gamma}$ are all positive. Also, let $a_{u}+m / 2-p / 2>0, a_{x}+$ $m / 2-1>0$ and $a_{\eta}+n_{t} / 2-m / 2>0$ where $p=\operatorname{rank}\left(\boldsymbol{X}_{*}\right)$ and $\boldsymbol{X}_{*}=\left(\boldsymbol{x}_{1}^{T}, \ldots, \boldsymbol{x}_{m}^{T}\right)^{T}$. Then the joint posterior is proper.

Proof: The basic full parameter space is $\boldsymbol{\Omega}=\left\{\boldsymbol{\theta}, \boldsymbol{b}, \boldsymbol{\gamma}, \boldsymbol{x}, \mu_{x}, \sigma_{x}^{2}, \sigma_{e}^{2}, \sigma_{u}^{2}, \sigma_{\eta}^{2}, \sigma_{\gamma}^{2}\right\}$. Let

$$
\begin{aligned}
I= & \int \cdots \int p(\boldsymbol{\Omega} \mid \mathbf{y}, \mathbf{X}) d \mathbf{\Omega} \\
= & \int \cdots \int\left[\mathbf{Y} \mid \boldsymbol{\theta}, \sigma_{e}^{2}\right]\left[\boldsymbol{\theta} \mid \boldsymbol{b}, \boldsymbol{\gamma}, \boldsymbol{x}, \sigma_{u}^{2}, \mathbf{X}\right]\left[\mathbf{X} \mid \boldsymbol{x}, \sigma_{\eta}^{2}\right]\left[\boldsymbol{x} \mid \mu_{x}, \sigma_{x}^{2}\right] \\
& \times\left[\boldsymbol{\gamma} \mid \sigma_{\gamma}^{2}\right][\boldsymbol{b}]\left[\mu_{x}\right]\left[\sigma_{e}^{2}\right]\left[\sigma_{u}^{2}\right]\left[\sigma_{\eta}^{2}\right]\left[\sigma_{\gamma}^{2}\right]\left[\sigma_{x}^{2}\right] d \mathbf{\Omega} .
\end{aligned}
$$

To show the propriety of the joint posterior, we have to prove that $I \leq M$ where $M$ is any finite positive constant.

First, integrating with respect to $\mu_{x}$ based on $\exp \left[-1 /\left(2 \sigma_{x}^{2}\right) \sum_{i=1}^{m}\left(x_{i}-\bar{x}\right)^{2}\right] \leq 1$,

$$
\begin{aligned}
I_{\mu_{x}} & =\int\left[\boldsymbol{x} \mid \mu_{x}, \sigma_{x}^{2}\right]\left[\mu_{x}\right] d \mu_{x} \\
& =\left(\sigma_{x}^{2}\right)^{-\frac{m}{2}} \int \exp \left[-\frac{1}{2 \sigma_{x}^{2}} \sum_{i=1}^{m}\left(x_{i}-\mu_{x}\right)^{2}\right] d \mu_{x} \\
& =\left(\sigma_{x}^{2}\right)^{-\frac{m}{2}} \exp \left[-\frac{1}{2 \sigma_{x}^{2}} \sum_{i=1}^{m}\left(x_{i}-\bar{x}\right)^{2}\right] \int \exp \left[-\frac{1}{2 \sigma_{x}^{2}} m\left(\mu_{x}-\bar{x}\right)^{2}\right] d \mu_{x} \\
& \leq K_{1} \cdot\left(\sigma_{x}^{2}\right)^{-\frac{m-1}{2}}
\end{aligned}
$$

where $K_{1}$ is a constant. Second, integrating with respect to $\boldsymbol{b}$ with $\mathbf{w}^{T}\left(I-P_{\mathbf{X}_{*}}\right) \mathbf{w} \geq 0$,

$$
\begin{aligned}
I_{\boldsymbol{b}} & =\int\left[\boldsymbol{\theta} \mid \boldsymbol{b}, \boldsymbol{\gamma}, \boldsymbol{x}, \sigma_{u}^{2}, \mathbf{X}\right][\boldsymbol{b}] d \boldsymbol{b} \\
& =\left(\sigma_{u}^{2}\right)^{-\frac{m}{2}} \int \exp \left[-\frac{1}{2 \sigma_{u}^{2}} \sum_{i=1}^{m}\left(\theta_{i}-\boldsymbol{x}_{i}^{T} \boldsymbol{b}-\mathbf{z}_{i}^{T} \gamma\right)^{2}\right] d \boldsymbol{b} \\
& =\left(\sigma_{u}^{2}\right)^{-\frac{m}{2}} \int \exp \left[-\frac{1}{2 \sigma_{u}^{2}} \sum_{i=1}^{m}\left(w_{i}-\boldsymbol{x}_{i}^{T} \boldsymbol{b}\right)^{2}\right] d \boldsymbol{b} \\
& =\left(\sigma_{u}^{2}\right)^{-\frac{m}{2}} \int \exp \left\{-\frac{1}{2 \sigma_{u}^{2}} \mathbf{w}^{T}\left(I-P_{\mathbf{X}_{*}}\right) \mathbf{w}\right\} d \boldsymbol{b}\left(\sigma_{u}^{2}\right)^{\frac{2}{p}}\left|\mathbf{X}_{*}^{T} \mathbf{X}_{*}\right|^{-\frac{1}{2}}(2 \pi)^{\frac{m}{2}} \\
& \leq K_{2} \cdot\left(\sigma_{u}^{2}\right)^{-\frac{(m-p)}{2}} \cdot\left|\mathbf{X}_{*}^{T} \mathbf{X}_{*}\right|^{-\frac{1}{2}},
\end{aligned}
$$

where $K_{2}$ is a constant and $P_{\mathbf{X}_{*}}=\mathbf{X}_{*}\left(\mathbf{X}_{*}^{T} \mathbf{X}_{*}\right)^{-1} \mathbf{X}_{*}^{T}$. Next, we integrate with respect to $\boldsymbol{x}$ based on the method by Ghosh et al. (2006).

$$
\begin{aligned}
I_{\boldsymbol{x}} & =\int\left[\mathbf{X} \mid \boldsymbol{x}, \sigma_{\eta}^{2}\right]\left|\mathbf{X}_{*}^{T} \mathbf{X}_{*}\right|^{-\frac{1}{2}} d \boldsymbol{x} \\
& =\left(\sigma_{\eta}^{2}\right)^{-\frac{n_{t}}{2}} \exp \left[-\frac{1}{2 \sigma_{\eta}^{2}} \sum_{i=1}^{m} \sum_{j=1}^{n_{i}}\left(X_{i j}-\bar{X}_{i}\right)^{2}\right] \int\left|\mathbf{X}_{*}^{T} \mathbf{X}_{*}\right|^{-\frac{1}{2}} \exp \left[-\frac{1}{2 \sigma_{\eta}^{2}} \sum_{i=1}^{m} n_{i}\left(\bar{X}_{i}-x_{i}\right)^{2}\right] d \boldsymbol{x}
\end{aligned}
$$




$$
\begin{aligned}
& \leq K_{3}^{\prime} \cdot\left(\sigma_{\eta}^{2}\right)^{-\frac{n_{t}-m}{2}} \exp \left[-\frac{1}{2 \sigma_{\eta}^{2}} \sum_{i=1}^{m} \sum_{j=1}^{n_{i}}\left(X_{i j}-\bar{X}_{i}\right)^{2}\right] \\
& \leq K_{3} \cdot\left(\sigma_{\eta}^{2}\right)^{-\frac{n_{t}-m}{2}}
\end{aligned}
$$

where $K_{3}^{\prime}$ and $K_{3}$ are some constants. Now, integrating with respect to $\sigma_{u}^{2}, \sigma_{x}^{2}$ and $\sigma_{\eta}^{2}$, using gamma distribution,

$$
\begin{aligned}
& I_{\sigma_{u}^{2}}=\int\left(\sigma_{u}^{2}\right)^{-\frac{m-p}{2}}\left[\sigma_{u}^{2}\right] d \sigma_{u}^{2}=\int\left(\sigma_{u}^{2}\right)^{-\left(a_{u}+\frac{m}{2}-\frac{p}{2}\right)-1} \exp \left(-\frac{b_{u}}{\sigma_{u}^{2}}\right) d \sigma_{u}^{2}=K_{4}, \\
& I_{\sigma_{x}^{2}}=\int\left(\sigma_{x}^{2}\right)^{-\frac{m-1}{2}}\left[\sigma_{x}^{2}\right] d \sigma_{x}^{2}=\int\left(\sigma_{x}^{2}\right)^{-\left(a_{x}+\frac{m}{2}-1\right)-1} \exp \left(-\frac{b_{x}}{\sigma_{x}^{2}}\right) d \sigma_{x}^{2}=K_{5}, \\
& I_{\sigma_{\eta}^{2}}=\int\left(\sigma_{\eta}^{2}\right)^{-\frac{n_{t}-m}{2}}\left[\sigma_{\eta}^{2}\right] d \sigma_{\eta}^{2}=\int\left(\sigma_{\eta}^{2}\right)^{-\left(a_{\eta}+\frac{n_{t}}{2}-\frac{m}{2}\right)-1} \exp \left(-\frac{b_{\eta}}{\sigma_{\eta}^{2}}\right) d \sigma_{\eta}^{2}=K_{6},
\end{aligned}
$$

where $K_{4}, K_{5}$ and $K_{6}$ are some constants.

Combining (3.1)-(3.7), we have

$$
I \leq K_{1} K_{2} K_{3} K_{4} K_{5} K_{6} \int \cdots \int\left[\mathbf{y} \mid \boldsymbol{\theta}, \sigma_{e}^{2}\right]\left[\gamma \mid \sigma_{\gamma}^{2}\right]\left[\sigma_{e}^{2}\right]\left[\sigma_{\gamma}^{2}\right] d \mathbf{\Omega}^{*},
$$

where $\boldsymbol{\Omega}^{*}=\left(\boldsymbol{\Omega}-\mu_{x}-\boldsymbol{b}-\boldsymbol{x}-\sigma_{u}^{2}-\sigma_{x}^{2}-\sigma_{\eta}^{2}\right)$. The posterior propriety is proved since the above integral would be finite due to all components of the integrand in (3.8) having proper distributions.

\subsection{Full conditional distributions}

To fit the model, we use MCMC integration technique for the implementation of the Bayesian procedure. The M-H algorithm and Gibbs sampler are used because $x_{i}$ has not full conditional distribution of closed form. We generate samples from the full conditional distribution of each $\boldsymbol{\theta}, \boldsymbol{b}, \boldsymbol{\gamma}, \boldsymbol{x}, \mu_{x}, \sigma_{x}^{2}$, $\sigma_{e}^{2}, \sigma_{u}^{2}, \sigma_{\eta}^{2}$ and $\sigma_{\gamma}^{2}$ given the remaining parameters and the data. Gibbs sampling and M-H algorithm are based on the following full conditional distributions:

(1) $\left[\theta_{i} \mid \boldsymbol{b}, \boldsymbol{\gamma}, \boldsymbol{x}, \mu_{x}, \sigma_{x}^{2}, \sigma_{e}^{2}, \sigma_{u}^{2}, \sigma_{\gamma}^{2}, \sigma_{\eta}^{2}, \boldsymbol{X}, \boldsymbol{y}\right] \stackrel{i i d}{\sim} N\left[\left(1-C_{i}\right) \bar{y}_{i}+C_{i}\left(\boldsymbol{x}_{i}^{T} \boldsymbol{b}+\boldsymbol{z}_{i}^{T} \gamma\right), \frac{\sigma_{e}^{2}}{n_{i}}\left(1-C_{i}\right)\right]$,

where $C_{i}=\sigma_{e}^{2} /\left(\sigma_{e}^{2}+n_{i} \sigma_{u}^{2}\right)$;

(2) $\left[\boldsymbol{b} \mid \boldsymbol{\theta}, \boldsymbol{\gamma}, \boldsymbol{x}, \mu_{x}, \sigma_{x}^{2}, \sigma_{e}^{2}, \sigma_{u}^{2}, \sigma_{\gamma}^{2}, \sigma_{\eta}^{2}, \boldsymbol{X}, \boldsymbol{y}\right] \sim N\left[\left(\boldsymbol{X}_{*}^{T} \boldsymbol{X}_{*}\right)^{-1} \boldsymbol{X}_{*}^{T} \boldsymbol{w}, \sigma_{u}^{2}\left(\boldsymbol{X}_{*}^{T} \boldsymbol{X}_{*}\right)^{-1}\right]$,

where $\boldsymbol{X}_{*}=\left(\boldsymbol{x}_{1}^{T}, \ldots, \boldsymbol{x}_{m}^{T}\right)^{T}, \mathbf{w}=\left(w_{1}, \ldots, w_{m}\right)^{T}, w_{i}=\theta_{i}-\boldsymbol{z}_{i}^{T} \gamma$;

(3) $\left[\gamma \mid \boldsymbol{\theta}, \boldsymbol{b}, \boldsymbol{x}, \mu_{x}, \sigma_{x}^{2}, \sigma_{e}^{2}, \sigma_{u}^{2}, \sigma_{\gamma}^{2}, \sigma_{\eta}^{2}, \boldsymbol{X}, \boldsymbol{y}\right] \sim N\left[\left(\frac{\boldsymbol{Z}_{*}^{T} \boldsymbol{Z}_{*}}{\sigma_{u}^{2}}+\frac{I}{\sigma_{\gamma}^{2}}\right)^{-1} \frac{\boldsymbol{Z}_{*}^{T}}{\sigma_{u}^{2}} \mathbf{t},\left(\frac{\boldsymbol{Z}_{*}^{T} \boldsymbol{Z}_{*}}{\sigma_{u}^{2}}+\frac{I}{\sigma_{\gamma}^{2}}\right)^{-1}\right]$, where $\mathbf{Z}_{*}=\left(\begin{array}{ccc}\left|x_{1}-\tau_{1}\right| & \cdots & \left|x_{1}-\tau_{k}\right| \\ \vdots & \ddots & \vdots \\ \left|x_{m}-\tau_{1}\right| & \cdots & \left|x_{m}-\tau_{k}\right|\end{array}\right), \mathbf{t}=\left(t_{1}, \ldots, t_{m}\right)^{T}, t_{i}=\theta_{i}-\boldsymbol{x}_{i}^{T} \boldsymbol{b} ;$ 
(4) $\left[x_{i} \mid \boldsymbol{\theta}, \boldsymbol{b}, \boldsymbol{\gamma}, \mu_{x}, \sigma_{x}^{2}, \sigma_{e}^{2}, \sigma_{u}^{2}, \sigma_{\gamma}^{2}, \sigma_{\eta}^{2}, \boldsymbol{X}, \boldsymbol{y}\right]$ $\stackrel{i i d}{\sim} \exp \left\{-\frac{1}{2 \sigma_{u}^{2}}\left(\theta_{i}-\boldsymbol{x}_{i}^{T} \boldsymbol{b}-z_{i}^{T} \gamma\right)^{2}\right\} N\left[\left(\sigma_{\eta}^{-2} n_{i}+\sigma_{x}^{-2}\right)^{-1}\left(\sigma_{\eta}^{-2} n_{i} \bar{X}_{i}+\sigma_{x}^{-2} \mu_{x}\right),\left(\sigma_{\eta}^{-2} n_{i}+\sigma_{x}^{-2}\right)^{-1}\right] ;$

(5) $\left[\mu_{x} \mid \boldsymbol{\theta}, \boldsymbol{b}, \boldsymbol{\gamma}, \boldsymbol{x}, \sigma_{x}^{2}, \sigma_{e}^{2}, \sigma_{u}^{2}, \sigma_{\gamma}^{2}, \sigma_{\eta}^{2}, \boldsymbol{X}, \boldsymbol{y}\right] \sim N\left(\bar{x}, \frac{\sigma_{x}^{2}}{m}\right)$;

(6) $\left[\sigma_{e}^{-2} \mid \boldsymbol{\theta}, \boldsymbol{b}, \boldsymbol{\gamma}, \boldsymbol{x}, \mu_{x}, \sigma_{x}^{2}, \sigma_{u}^{2}, \sigma_{\gamma}^{2}, \sigma_{\eta}^{2}, \boldsymbol{X}, \boldsymbol{y}\right] \sim G\left[\frac{n_{t}}{2}+a_{e}, \frac{1}{2} \sum_{i=1}^{m} \sum_{j=1}^{n_{i}}\left(y_{i j}-\theta_{i}\right)^{2}+b_{e}\right]$;

(7) $\left[\sigma_{u}^{-2} \mid \boldsymbol{\theta}, \boldsymbol{b}, \boldsymbol{\gamma}, \boldsymbol{x}, \mu_{x}, \sigma_{x}^{2}, \sigma_{e}^{2}, \sigma_{\gamma}^{2}, \sigma_{\eta}^{2}, \boldsymbol{X}, \boldsymbol{y}\right] \sim G\left[\frac{m}{2}+a_{u}, \frac{1}{2} \sum_{i=1}^{m}\left(\theta_{i}-\boldsymbol{x}_{i}^{T} \boldsymbol{b}-z_{i}^{T} \gamma\right)^{2}+b_{u}\right]$;

(8) $\left[\sigma_{\eta}^{-2} \mid \boldsymbol{\theta}, \boldsymbol{b}, \boldsymbol{\gamma}, \boldsymbol{x}, \mu_{x}, \sigma_{x}^{2}, \sigma_{e}^{2}, \sigma_{\gamma}^{2}, \sigma_{u}^{2}, \boldsymbol{X}, \boldsymbol{y}\right] \sim G\left[\frac{n_{t}}{2}+a_{\eta}, \frac{1}{2} \sum_{i=1}^{m} \sum_{j=1}^{n_{i}}\left(X_{i j}-x_{i}\right)^{2}+b_{\eta}\right]$;

(9) $\left[\sigma_{\gamma}^{-2} \mid \boldsymbol{\theta}, \boldsymbol{b}, \boldsymbol{\gamma}, \boldsymbol{x}, \mu_{x}, \sigma_{x}^{2}, \sigma_{e}^{2}, \sigma_{u}^{2}, \sigma_{\eta}^{2}, \boldsymbol{X}, \boldsymbol{y}\right] \sim G\left[\frac{k}{2}+a_{\gamma}, \frac{1}{2} \boldsymbol{\gamma}^{T} \boldsymbol{\gamma}+b_{\gamma}\right]$;

(10) $\left[\sigma_{x}^{-2} \mid \boldsymbol{\theta}, \boldsymbol{b}, \boldsymbol{\gamma}, \boldsymbol{x}, \mu_{x}, \sigma_{\gamma}^{2}, \sigma_{e}^{2}, \sigma_{u}^{2}, \sigma_{\eta}^{2}, \boldsymbol{X}, \boldsymbol{y}\right] \sim G\left[\frac{m}{2}+a_{x}, \frac{1}{2} \sum_{i=1}^{m}\left(x_{i}-\mu_{x}\right)^{2}+b_{x}\right]$.

Our target of inference is to estimate $\left\{\theta_{i}, i=1, \ldots, m\right\}$. We run $L(\geq 2)$ chains and $2 d$ iterations for each chain. To reduce the effect of the starting distributions, the first $d$ iterations of each chain are discarded and posterior summaries are calculated based on remaining $d$ iterates. The HB estimators for small area means is approximated by

$$
\begin{aligned}
E\left(\theta_{i} \mid \boldsymbol{X}, \boldsymbol{y}\right) & =E\left[E\left(\theta_{i} \mid \boldsymbol{b}, \boldsymbol{\gamma}, \boldsymbol{x}, \mu_{x}, \sigma_{x}^{2}, \sigma_{e}^{2}, \sigma_{u}^{2}, \sigma_{\gamma}^{2}, \sigma_{\eta}^{2}, \boldsymbol{X}, \boldsymbol{y}\right)\right] \\
& \simeq\left(L d^{-1}\right) \sum_{l=1}^{L} \sum_{r=d+1}^{2 d}\left[\left(1-C_{i}^{(l r)}\right) \bar{y}_{i}+C_{i}^{(l r)}\left(\boldsymbol{x}_{i}^{T(l r)} \boldsymbol{b}^{(l r)}+\mathbf{z}_{i}^{T(l r)} \boldsymbol{\gamma}^{(l r)}\right)\right],
\end{aligned}
$$

and the posterior variance is estimated by

$$
\begin{aligned}
V\left(\theta_{i} \mid \boldsymbol{X}, \boldsymbol{y}\right)= & E\left[V\left(\theta_{i} \mid \boldsymbol{b}, \boldsymbol{\gamma}, \boldsymbol{x}, \mu_{x}, \sigma_{x}^{2}, \sigma_{e}^{2}, \sigma_{u}^{2}, \sigma_{\gamma}^{2}, \sigma_{\eta}^{2}, \boldsymbol{X}, \boldsymbol{y}\right)\right] \\
& +V\left[E\left(\theta_{i} \mid \boldsymbol{b}, \boldsymbol{\gamma}, \boldsymbol{x}, \mu_{x}, \sigma_{x}^{2}, \sigma_{e}^{2}, \sigma_{u}^{2}, \sigma_{\gamma}^{2}, \sigma_{\eta}^{2}, \mathbf{X}, \mathbf{y}\right)\right] \\
\simeq & \left(L d^{-1}\right) \sum_{l=1}^{L} \sum_{r=d+1}^{2 d}\left(\frac{\sigma_{e}^{2(l r)}}{n_{i}}\left(1-C_{i}^{(l r)}\right)\right) \\
& +\left(L d^{-1}\right) \sum_{l=1}^{L} \sum_{r=d+1}^{2 d}\left[\left(1-C_{i}^{(l r)}\right) \bar{y}_{i}+C_{i}^{(l r)}\left(\boldsymbol{x}_{i}^{T(l r)} \boldsymbol{b}^{(l r)}+\mathbf{z}_{i}^{T(l r)} \boldsymbol{\gamma}^{(l r)}\right)\right]^{2} \\
& -\left[E\left(\theta_{i} \mid \boldsymbol{X}, \boldsymbol{y}\right)\right]^{2} .
\end{aligned}
$$




\subsection{Convergence and model adequacy}

We implement MCMC chains and monitored the convergence of the MCMC following general guidelines based on $\sqrt{\hat{R}_{i}}$ (Gelman and Rubin, 1992) as the estimator of PSRF. This is given by

$$
\sqrt{\hat{R}_{i}}=\sqrt{\frac{\widehat{\operatorname{var}}\left(\theta_{i}\right)}{W_{i}}}
$$

where $\widehat{\operatorname{var}}\left(\theta_{i}\right)=(1-1 / n) W_{i}+(1 / n) B_{i}, W_{i}$ and $B_{i}$ are within chain variance and between chain variance, respectively. If $\sqrt{\hat{R}_{i}}$ is close to 1 for all $\theta_{i}$, it implies that the MCMC chains are convergent.

We calculate the posterior predictive $p$-value (Meng, 1994) to check the model adequacy. The model is better supported by the data if the posterior predictive $p$-value $(p)$ under the model is close to 0.5 .

$$
p=(L d)^{-1} \sum_{l=1}^{L} \sum_{r=d+1}^{2 d} I\left\{d\left(\mathbf{y}^{(l r)}, \boldsymbol{\theta}^{(l r)}\right) \geq d\left(\mathbf{y}_{o b s}, \boldsymbol{\theta}^{(l r)}\right)\right\},
$$

where $I(\cdot)$ is indicator function and $d(\mathbf{y}, \boldsymbol{\theta})$ is the posterior discrepancy measure.

\section{Simulation Studies}

\subsection{Computational details}

The key feature of our implementation is the use of a semiparametric regression model with a RBF instead of TPBF. To compare the performance between these two choices in the model, we consider two functions (a slowly-varying smooth function and a function with a sharp peak).

Example 1. The true function is

$$
f(x)=5+3 x+4 x^{2}, \quad x \in[-2,2] .
$$

Example 2. The true function is

$$
f(x)=\sin (x)+0.5 \exp \left(-3 x^{2}\right), \quad x \in[-2,2] .
$$

To take simulated data, at first, we sequentially generate $x_{i}(i=1, \ldots, 12)$ on $[-2,2]$ and then we generate $X_{i j}$ from $x_{i}$ with error $\eta_{i j} \sim N\left(0,0.3^{2}\right)$. And $\theta_{i}$ are generated from $x_{i}$ with random effect $u_{i} \sim N\left(0,0.1^{2}\right)$ for each function. Lastly, $y_{i j}$ are generated from $\theta_{i}$ with errors $e_{i j} \sim N\left(0,2^{2}\right)$ and $e_{i j} \sim N\left(0,0.3^{2}\right)$ for each examples. We run three independent chains with runs of length 5,000 following burn-ins of 2,500. We set all 1.0 for all hyperparameters $a_{e}, b_{e}, a_{u}, b_{u}, a_{\eta}, b_{\eta}, a_{\eta}, b_{\eta}, a_{x}$ and $b_{x}$. Notice that the results are not sensitive to other values for the hyperparameters. We then take the average of the squared differences of the estimators from the true mean (TM) over the 100 independent simulations and take their squared roots to obtain the root mean squared errors (RMSE) to compare the performance, where

$$
\mathrm{RMSE}_{i}=\sqrt{\sum_{k=1}^{100} \frac{\left(\theta_{i}^{(k)}-\hat{\theta}_{i}^{(k)}\right)^{2}}{100}}
$$


Table 1: Estimates of small area means for Example 1

\begin{tabular}{|c|c|c|c|c|c|c|c|c|c|c|}
\hline \multirow{2}{*}{$i$} & \multirow[b]{2}{*}{$n_{i}$} & \multirow{2}{*}{ TM } & \multicolumn{4}{|c|}{ Mean with TPBF } & \multicolumn{4}{|c|}{ Mean with RBF } \\
\hline & & & $K=1$ & $K=3$ & $K=5$ & $K=7$ & $K=1$ & $K=3$ & $K=5$ & $K=7$ \\
\hline 1 & 5 & 15.015 & 14.441 & 14.903 & 14.890 & 14.884 & 14.443 & 14.909 & 14.910 & 14.908 \\
\hline 2 & 10 & 10.847 & 10.829 & 10.804 & 10.778 & 10.784 & 10.831 & 10.805 & 10.780 & 10.782 \\
\hline 3 & 5 & 7.656 & 8.183 & 7.744 & 7.818 & 7.822 & 8.184 & 7.744 & 7.806 & 7.815 \\
\hline 4 & 7 & 5.587 & 6.006 & 5.570 & 5.668 & 5.652 & 6.006 & 5.569 & 5.661 & 5.645 \\
\hline 5 & 8 & 4.563 & 4.727 & 4.742 & 4.594 & 4.606 & 4.726 & 4.739 & 4.590 & 4.603 \\
\hline 6 & 8 & 4.580 & 4.231 & 4.599 & 4.635 & 4.632 & 4.229 & 4.597 & 4.633 & 4.629 \\
\hline 7 & 7 & 5.658 & 5.385 & 5.635 & 5.692 & 5.693 & 5.384 & 5.634 & 5.692 & 5.692 \\
\hline 8 & 9 & 7.854 & 8.046 & 8.059 & 7.998 & 8.003 & 8.045 & 8.058 & 7.999 & 8.002 \\
\hline 9 & 8 & 11.040 & 11.292 & 11.074 & 11.145 & 11.121 & 11.292 & 11.077 & 11.145 & 11.122 \\
\hline 10 & 7 & 15.264 & 15.382 & 15.234 & 15.213 & 15.238 & 15.383 & 15.235 & 15.215 & 15.237 \\
\hline 11 & 7 & 20.674 & 20.602 & 20.604 & 20.591 & 20.590 & 20.599 & 20.605 & 20.595 & 20.591 \\
\hline 12 & 5 & 26.987 & 26.648 & 26.845 & 26.849 & 26.848 & 26.651 & 26.844 & 26.853 & 26.855 \\
\hline
\end{tabular}

$\mathrm{TM}=$ true mean; TPBF = truncated polynomial basis functions; RBF = radial basis functions.

Table 2: RMSE for Example 1

\begin{tabular}{|c|c|c|c|c|c|c|c|c|c|}
\hline \multirow{2}{*}{$i$} & \multirow[b]{2}{*}{$n_{i}$} & \multicolumn{4}{|c|}{ RMSE with TPBF } & \multicolumn{4}{|c|}{ RMSE with RBF } \\
\hline & & $K=1$ & $K=3$ & $K=5$ & $K=7$ & $K=1$ & $K=3$ & $K=5$ & $K=7$ \\
\hline 1 & 5 & 1.022 & 0.881 & 0.920 & 0.942 & 1.023 & 0.871 & 0.910 & 0.918 \\
\hline 2 & 10 & 0.548 & 0.560 & 0.580 & 0.579 & 0.548 & 0.560 & 0.580 & 0.580 \\
\hline 3 & 5 & 0.828 & 0.708 & 0.678 & 0.700 & 0.828 & 0.708 & 0.684 & 0.702 \\
\hline 4 & 7 & 0.731 & 0.593 & 0.602 & 0.590 & 0.730 & 0.592 & 0.600 & 0.590 \\
\hline 5 & 8 & 0.504 & 0.454 & 0.445 & 0.444 & 0.505 & 0.453 & 0.444 & 0.444 \\
\hline 6 & 8 & 0.612 & 0.441 & 0.446 & 0.448 & 0.612 & 0.442 & 0.445 & 0.449 \\
\hline 7 & 7 & 0.701 & 0.574 & 0.561 & 0.559 & 0.702 & 0.576 & 0.561 & 0.559 \\
\hline 8 & 9 & 0.656 & 0.617 & 0.609 & 0.621 & 0.656 & 0.620 & 0.609 & 0.621 \\
\hline 9 & 8 & 0.704 & 0.659 & 0.665 & 0.662 & 0.704 & 0.657 & 0.665 & 0.662 \\
\hline 10 & 7 & 0.754 & 0.767 & 0.771 & 0.762 & 0.754 & 0.771 & 0.772 & 0.766 \\
\hline 11 & 7 & 0.774 & 0.784 & 0.793 & 0.802 & 0.773 & 0.784 & 0.795 & 0.804 \\
\hline 12 & 5 & 0.887 & 0.853 & 0.863 & 0.859 & 0.885 & 0.853 & 0.858 & 0.856 \\
\hline \multicolumn{2}{|c|}{ Overall } & 8.721 & 7.891 & 7.933 & 7.968 & 8.720 & 7.887 & 7.923 & 7.951 \\
\hline
\end{tabular}

RMSE = root mean square error; TPBF = truncated polynomial basis functions; $\mathrm{RBF}$ = radial basis functions.

\subsection{Results}

In all cases, $\hat{R}_{i} \simeq 1$ for all $\theta_{i}$ and $p \simeq 0.5$ for all simulation data and models. The detailed results are reported in Tables 1-4. We report the sample sizes, TM and small area means estimates with two basis functions as well as RMSE for each strata in Examples 1 and 2. In Example 1, we can see that the model with RBF provides minimal improvement for all number of knots based on the overall RMSE; however, the difference is only a third decimal point. The performance of the model with RBF is also very similar with TPBF in Example 2. Additionally, the case of three knots has the smallest RMSE for both basis functions. Figure 1 shows the one result $(K=3$ case) for Examples 1 and 2 that the real line $(-)$, dashed line $(---)$, and dotted line $(\cdots \cdots)$ are the true function and the estimates with TPBS and RBF, respectively.

\section{Application}

We conducted the analysis based on a real data. We used LANDSAT (Land observatory satellites) data by Battese et al. (1988), hereafter BHF, for analysis. BHF was made available by Tobias Schoch with the R package "rsae". This data on the areas under corn and soybeans (reported in hectares) in the 37 segments of the 12 counties (north-central Iowa) were determined by USDA Statistical Reporting 
Table 3: Estimates of small area means for Example 2

\begin{tabular}{|c|c|c|c|c|c|c|c|c|c|c|}
\hline \multirow{2}{*}{$i$} & \multirow[b]{2}{*}{$n_{i}$} & \multirow{2}{*}{$\mathrm{TM}$} & \multicolumn{4}{|c|}{ Mean with TPBF } & \multicolumn{4}{|c|}{ Mean with RBF } \\
\hline & & & $K=1$ & $K=3$ & $K=5$ & $K=7$ & $K=1$ & $K=3$ & $K=5$ & $K=7$ \\
\hline 1 & 5 & -0.895 & -0.896 & -0.883 & -0.882 & -0.881 & -0.899 & -0.877 & -0.876 & -0.874 \\
\hline 2 & 10 & -0.989 & -1.000 & -0.998 & -0.999 & -0.999 & -1.001 & -0.999 & -1.000 & -1.000 \\
\hline 3 & 5 & -0.941 & -0.903 & -0.911 & -0.910 & -0.912 & -0.904 & -0.918 & -0.916 & -0.918 \\
\hline 4 & 7 & -0.785 & -0.766 & -0.775 & -0.774 & -0.776 & -0.766 & -0.781 & -0.780 & -0.782 \\
\hline 5 & 8 & -0.520 & -0.497 & -0.502 & -0.504 & -0.504 & -0.496 & -0.504 & -0.507 & -0.507 \\
\hline 6 & 8 & 0.568 & 0.550 & 0.549 & 0.548 & 0.548 & 0.552 & 0.552 & 0.551 & 0.552 \\
\hline 7 & 7 & 0.924 & 0.890 & 0.892 & 0.893 & 0.894 & 0.893 & 0.898 & 0.899 & 0.900 \\
\hline 8 & 9 & 0.517 & 0.541 & 0.545 & 0.546 & 0.547 & 0.542 & 0.548 & 0.549 & 0.549 \\
\hline 9 & 8 & 0.787 & 0.790 & 0.796 & 0.796 & 0.797 & 0.791 & 0.797 & 0.797 & 0.797 \\
\hline 10 & 7 & 0.957 & 0.940 & 0.943 & 0.943 & 0.943 & 0.939 & 0.943 & 0.943 & 0.943 \\
\hline 11 & 7 & 0.993 & 0.983 & 0.982 & 0.982 & 0.982 & 0.982 & 0.981 & 0.981 & 0.981 \\
\hline 12 & 5 & 0.897 & 0.931 & 0.924 & 0.922 & 0.921 & 0.928 & 0.920 & 0.918 & 0.918 \\
\hline
\end{tabular}

TM = true mean; TPBF = truncated polynomial basis functions; RBF = radial basis functions.

Table 4: RMSE for Example 2

\begin{tabular}{|c|c|c|c|c|c|c|c|c|c|}
\hline \multirow{2}{*}{$i$} & \multirow{2}{*}{$n_{i}$} & \multicolumn{4}{|c|}{ RMSE with TPBF } & \multicolumn{4}{|c|}{ RMSE with RBF } \\
\hline & & $K=1$ & $K=3$ & $K=5$ & $K=7$ & $K=1$ & $K=3$ & $K=5$ & $K=7$ \\
\hline 1 & 5 & 0.138 & 0.139 & 0.140 & 0.141 & 0.138 & 0.140 & 0.141 & 0.142 \\
\hline 2 & 10 & 0.095 & 0.095 & 0.095 & 0.095 & 0.096 & 0.095 & 0.096 & 0.096 \\
\hline 3 & 5 & 0.127 & 0.125 & 0.125 & 0.125 & 0.127 & 0.124 & 0.125 & 0.124 \\
\hline 4 & 7 & 0.111 & 0.110 & 0.111 & 0.111 & 0.111 & 0.110 & 0.110 & 0.110 \\
\hline 5 & 8 & 0.089 & 0.088 & 0.088 & 0.088 & 0.089 & 0.087 & 0.087 & 0.088 \\
\hline 6 & 8 & 0.091 & 0.092 & 0.092 & 0.092 & 0.091 & 0.092 & 0.092 & 0.092 \\
\hline 7 & 7 & 0.111 & 0.111 & 0.111 & 0.11 & 0.111 & 0.109 & 0.109 & 0.109 \\
\hline 8 & 9 & 0.104 & 0.105 & 0.106 & 0.107 & 0.105 & 0.107 & 0.107 & 0.108 \\
\hline 9 & 8 & 0.108 & 0.109 & 0.109 & 0.109 & 0.108 & 0.109 & 0.109 & 0.110 \\
\hline 10 & 7 & 0.122 & 0.122 & 0.123 & 0.123 & 0.123 & 0.123 & 0.124 & 0.123 \\
\hline 11 & 7 & 0.123 & 0.123 & 0.124 & 0.124 & 0.123 & 0.124 & 0.124 & 0.125 \\
\hline 12 & 5 & 0.131 & 0.131 & 0.131 & 0.131 & 0.130 & 0.130 & 0.131 & 0.131 \\
\hline \multicolumn{2}{|c|}{ Overall } & 1.350 & 1.350 & 1.355 & 1.356 & 1.352 & 1.350 & 1.355 & 1.358 \\
\hline
\end{tabular}

RMSE $=$ root mean square error; $\mathrm{TPBF}=$ truncated polynomial basis functions; $\mathrm{RBF}=$ radial basis functions.

\section{Example 1}

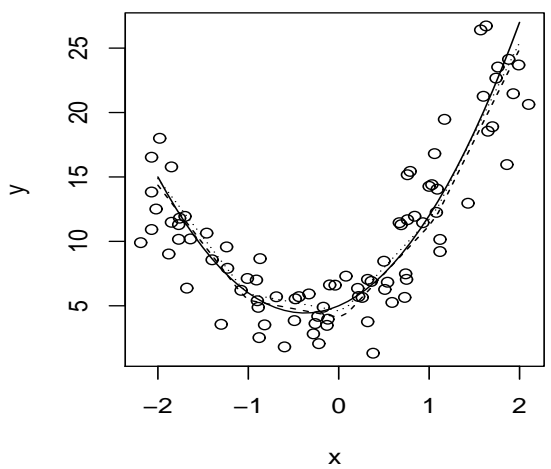

Example 2

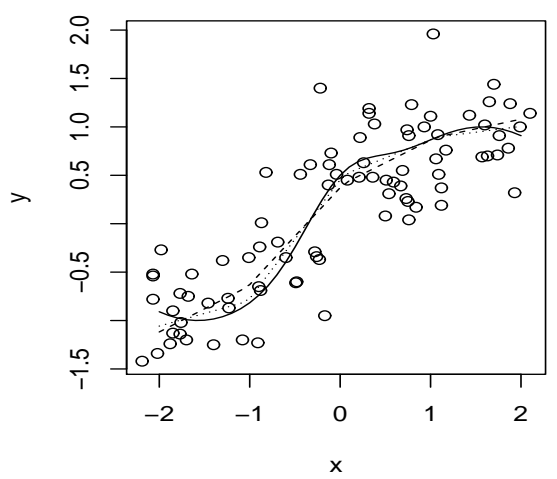

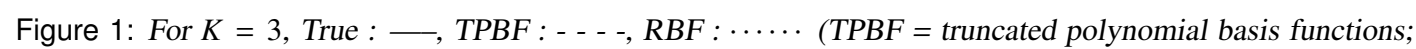
$R B F=$ radial basis functions). 


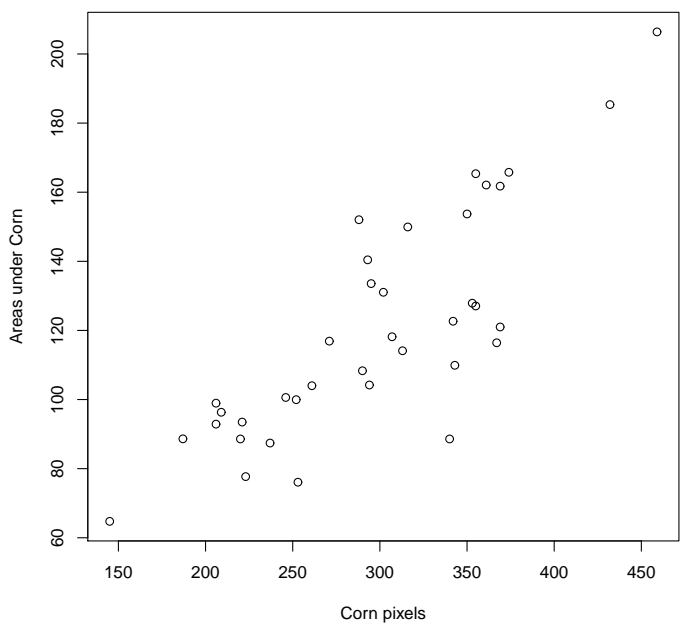

Figure 2: Scatter plot for areas under corn and corn pixels.

Table 5: Comparative data analyses for the LANDSAT data

\begin{tabular}{|c|c|c|c|c|c|c|c|c|c|c|c|c|c|}
\hline \multirow{3}{*}{ Counties } & \multirow{3}{*}{$n_{i}$} & \multicolumn{4}{|c|}{ TPBF } & & & \multicolumn{6}{|c|}{ RBF } \\
\hline & & \multicolumn{2}{|c|}{$K=1$} & \multicolumn{2}{|c|}{$K=3$} & \multicolumn{2}{|c|}{$K=5$} & \multicolumn{2}{|c|}{$K=1$} & \multicolumn{2}{|c|}{$K=3$} & \multicolumn{2}{|c|}{$K=5$} \\
\hline & & Est. & S.E. & Est. & S.E. & Est. & S.E. & Est. & S.E. & Est. & S.E. & Est. & S.E. \\
\hline erro Gordo & 1 & 151.039 & 9.779 & 142.155 & 14.020 & 142.738 & 14.477 & 159.396 & 9.188 & 155.336 & 10.114 & 149.823 & 12.915 \\
\hline Hamilton & 1 & 98.541 & 10.431 & 79.712 & 36.185 & 93.086 & 16.925 & 104.270 & 12.058 & 95.364 & 18.822 & 90.516 & 44.626 \\
\hline & 1 & 107.205 & 6.457 & 24.905 & 19.296 & 1.662 & 12.022 & 7.216 & 5.570 & 106.394 & 11.703 & 105.727 & 11.598 \\
\hline Hum & 2 & 162.104 & 13.115 & 142.849 & 19.889 & 141.193 & 19.516 & 174.935 & 12.647 & 166.024 & 16.310 & 157.566 & 18.405 \\
\hline Fra & 3 & 35.980 & 6.150 & 136.407 & 11.033 & 39.456 & 10.942 & 8.206 & 5.754 & 139.369 & 7.814 & 140.704 & 10.729 \\
\hline Poca & 3 & 101.258 & 8.810 & 95.917 & 20.708 & 106.723 & 12.125 & 105.209 & 9.663 & 98.983 & 12.783 & 105.379 & 14.144 \\
\hline Wir & 3 & 03 & 6 & 3.916 & 99 & .501 & 9.3 & .005 & 06 & 38 & 7.386 & 110.107 & 7.626 \\
\hline V & 3 & 141.251 & 7.130 & 140.075 & 13.581 & 141.530 & 11.196 & 145.761 & 6.684 & 145.891 & 8.795 & 143.483 & 10.436 \\
\hline We & 4 & 109.644 & 5.966 & 121.833 & 13.821 & 115.458 & 11.474 & 108.218 & 4.954 & 108.286 & 8.621 & 106.516 & 12.537 \\
\hline $\mathrm{Ha}$ & 5 & 113.533 & 6.372 & 115.107 & 8.265 & 114.080 & 7.645 & 109.478 & 6.068 & 111.308 & 6.278 & 109.121 & 6.961 \\
\hline $\mathrm{Kc}$ & 5 & 123.925 & 5.763 & 120.675 & 8.176 & 121.354 & 9.577 & 121.904 & 5.806 & 123.941 & 6.233 & 123.089 & 6.105 \\
\hline Hardin & 6 & 113.980 & 6.398 & 15.231 & 8.915 & 115.162 & 8.506 & 109.705 & 6.240 & 111.664 & 6.398 & 109.471 & 6.935 \\
\hline$p$-value & & 0.4 & & 0.4 & & 0.4 & & & & 0.4 & & & \\
\hline
\end{tabular}

LANDSAT = land observatory satellites; TPBF = truncated polynomial basis functions; RBF = radial basis functions.

Service staff consists of 37 observations and 10 variables. This paper considers a prediction of areas under corn that uses soya corn pixels only as a measurement error covariate. Figure 2 is the scatter plot for areas under corn and corn pixels; consequently, we can view it is a little non-linear pattern.

We use same settings such as simulation studies. In our all case, $\sqrt{\hat{R}} \simeq 1$ for all $\theta_{i}$ and we report the sample sizes, estimates, standard errors (s.e.) and the posterior predictive $p$-value for each case in Table 5. The models with five knots TPBF and one knot RBF are better based on $p$-value and these two models have similar model adequacy.

\section{Concluding Remarks}

We developed a semiparametric small area regression model with radial basis functions instead of truncated polynomial basis functions under a structural measurement error model with knots on a grid of equally spaced sample quantiles of the covariate. Numerical studies show that estimates of small 
area means seem similar to estimates based on truncated polynomial basis functions.

We can extend our model in various way. First, we considered normal outcome and covariate with the measurement error in this paper. We develop generalized versions for the measurement error problem in binomial or Poisson outcome cases. Second, we only considered measurement error in covariate without measurement error in outcome variable. We can consider measurement error in both covariate and outcome variables. Third, the results depend on the number and location of knots. The selection of knots is always a subjective and tricky issue in these kinds of problems. The complete underlying pattern may not be captured properly and result in a biased fit if too few or too many knots are used; therefore, we will handle semiparametric Bayesian estimation with free knots in future research.

\section{References}

Battese, G. E., Harter, R. M. and Fuller, W. A. (1988). An error-components model for prediction of county crop areas using survey and satellite data, Journal of the American Statistical Association, 83, 28-36.

Bhadra, D., Ghosh, M. and Kim, D. (2013). Estimation of median household income for small areas: A Bayesian semiparametric approach, Calcutta Statistical Association Bulletin, 64, 115-142.

Fuller, W. A. (1987). Measurement Error Models, John Wiley \& Sons, New York.

Ghosh, M., Sinha, K. and Kim, D. (2006). Empirical and hierarchical Bayesian estimation in finite population sampling under structural measurement error models, Scandinavian Journal of Statistics, 33, 591-608.

Gelman, A. E. and Rubin, D. B. (1992). Inference from iterative simulation using multiple sequences, Statistical Science, 7, 457-472.

Hwang, J. S. and Kim, D. H. (2010). Semiparametric Bayesian estimation under structural measurement error model, Communications of the Korean Statistical Society, 17, 551-560.

Meng, X. L. (1994). Posterior predictive p-values. Annals of Statistics, 22, 1142-1160.

Opsomer, J. D., Claeskens, G., Ranalli, M. G., Kauermann, G. and Breidt, F. J. (2008). Nonparametric small area estimation using penalized spline regression, Journal of the Royal Statistical Society Series B (Statistical Methodology), 70, 265-286.

Rao, J. N. K. (2003). Small Area Estimation, John Wiley \& Sons, New York.

Ruppert, D., Wand, M. P. and Carroll, R. J. (2003). Semiparametric Regression, Cambridge University Press, Cambridge. 